\title{
Zwischen Theorie und Wirklichkeit: Römische Sicherheitsgesetze und ihre Realisierung
}

\author{
Peter Herz
}

Wenn man einem griechischen Intellektuellen des 2. Jahrhunderts n. Chr. wie Aelius Aristides unbedingt Glauben schenken möchte, dann muss das Imperium Romanum während seiner Lebenszeit ein wahres Paradies auf Erden gewesen sein. So macht uns Aristides in seinem Panegyricus Eis Romen mit einem Imperium Romanum vertraut, dessen Lebensumstände eher an das Goldene Zeitalter der Mythen erinnern als dass es einen Platz in der Realität hätte. ${ }^{1}$ Ausgangspunkt für meine Überlegungen sind die beiden folgenden Abschnitte aus dieser großen Rede:

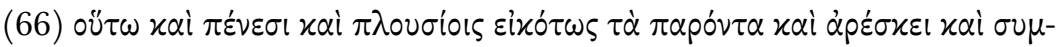

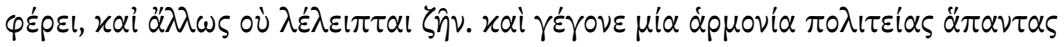

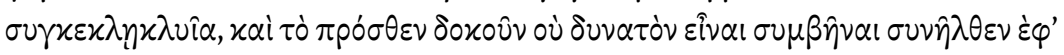

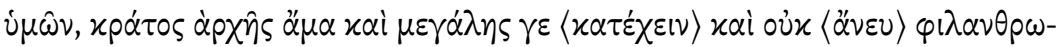

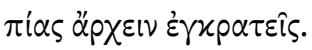

(66) So sind die bestehenden Verhältnisse naturgemäß sowohl für die Armen als auch die Reichen befriedigend und nützlich, und eine andere Art zu leben gibt es nicht. So hat sich eine einzige Harmonie staatlicher Ordnung entwickelt, die alle einschließt, und was früher offensichtlich nicht zusammentreffen konnte, hat sich unter euch vereinigt: Ihr seid fähig, zugleich die Macht über ein Reich, und dazu ein gewaltiges, auszuüben und es nicht ohne Menschenfreundlichkeit $(\varphi \imath \lambda \alpha \nu \theta \rho \omega \pi i \alpha) \mathrm{zu}$ beherrschen.

1 R. Klein, Die Romrede des Aelius Aristides. Einführung (Darmstadt 1981) und ders., Die Romrede des Aelius Aristides, herausgegeben, übersetzt und mit Erläuterungen versehen (Darmstadt 1983); P. Desideri, F. Fontanella, Elio Aristide e la legittimazione greca dell'impero Romano (Bologna 2013). Immer noch grundlegend J.H. Oliver, The Ruling Power. A Study of the Roman Empire in the Second Century After Christ Through the Roman Oration of Aelius Aristides (Philadelphia 1953) (Paperback Nachdruck o. O. 2013). 


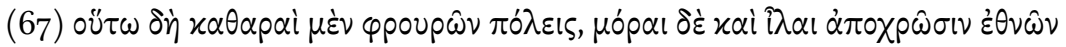

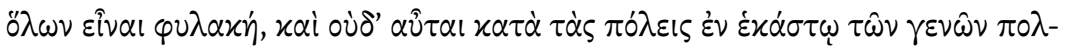

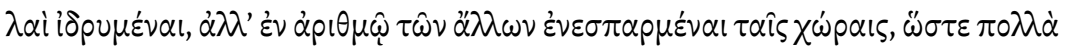

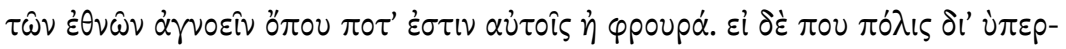

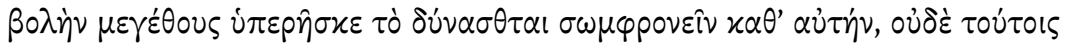

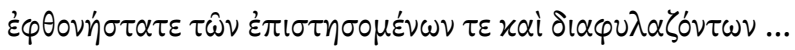

(67) So sind die Städte frei von Besatzungen, Kohorten und Reiterabteilungen genügen zur Beaufsichtigung ganzer Provinzen, und nicht einmal jene sind in größerer Menge auf die Städte der einzelnen Stämme verteilt, sondern sie leben entsprechend der übrigen Bevölkerung verstreut im Land, so dass viele der Provinzen gar nicht wissen, wo ihre Besatzung steht. Wenn aber irgendwo eine Stadt wegen ihrer übermäßigen Größe aus eigener Kraft die Ordnung nicht aufrechterhalten kann, so habt ihr auch dieser die Leute, die sie regieren und schützen sollen, nicht vorenthalten ...

In den Augen eines Aelius Aristides repräsentierte die hier geschilderte Ordnung der Welt einen geradezu paradiesischen Zustand. Und, ohne dass er dies ausdrücklich ausspricht, wird sehr deutlich, dass es sich dabei in seinen Augen um einen Zustand handelt, an dem man tunlichst nicht rühren sollte. Zu den bedeutenden Denkern, die Aelius Aristides Glauben schenkten und daher diesem phantastischen Bild etwas auf den Leim gegangen sind, gehörte auch keine geringere Persönlichkeit wie Edward Gibbon, der u.a. auf der Grundlage eines Aelius Aristides die Epoche der Antonine zu einer der glücklichsten Epochen in der Geschichte der Menschheit deklarierte. ${ }^{2}$

Es sollte aber zum alltäglichen Handwerkszeug eines Historikers gehören, solche globalen Aussagen, vor allem wenn sie ein so strahlendes Bild einer Epoche zu vermitteln suchen, kritisch zu hinterfragen und das von ihnen präsentierte Bild mit zusätzlichen Informationen zu konfrontieren, die von anderen Quellen bereitgestellt werden. Daher möchte ich dieses Bild zunächst mit einem Zeugnis aus dem Lykien des späten 2. Jh. konfrontieren:

Imperator Caesar Marcus Aurelius Commodus Antoninus Pius Felix Augustus Sarmaticus Germanicus maximus Britannicus, Sohn des divus Marcus Antoninus Augustus Germanicus Sarmaticus [esfolgt die gesamte

2 Ed. Gibbon, Verfall und Untergang des römischen Imperiums I, aus dem Englischen von M. Walter (Darmstadt 2016), 106. 
Abfolge der Vorfahren bis auf Kaiser Nerva], pontifex maximus, im 15. Jahr seiner tribunicia potestas, mit der 8. Imperatorischen Akklamation, zum sechstenmal consul, pater patriae, grüßt die Archonten und die boulé und den Rat der Bouboneoi.

Ich habe euch wegen eures Mutes und eurer Tapferkeit gelobt und habe den gemeinsamen Beschluß des lykischen Volkes angenommen, daß ihr mit einem solchen Mut zur Ergreifung der Räuber aufgebrochen seid, sie besiegt und einen Teil von ihnen getötet, einen anderen Teil lebendig gefangen genommen habt. Diesbezüglich hat euch das Koinon der Lykier richtig die passende Ehre erwiesen, euch noch eine Stimme dazuzuerteilen, wodurch ihr noch berühmter werden müsstet, und es hat auch die anderen mutiger gemacht zu solchen Heldentaten. Daher habe ich auch den Antrag des gemeinsamen Beschlusses bestätigt und euch erlaubt, fortan unter die Städte mit einem dreifachen Stimmrecht gezählt zu werden.

Die Gesandtschaft hatte Meleager, Sohn des Meleager, Enkel des Artemon, zweimal unternommen. Lebt wohl! ${ }^{3}$

Die Gemeinde von Bubon hatte also mit ihren eigenen Ressourcen erfolgreich den Versuch unternommen, nicht genauer charakterisierte Räuber zu verfolgen und auszuschalten. Die Gemeinde hatte damit in ihrer eigenen Verantwortung und wohl auch auf ihre eigenen Kosten eine Aufgabe geschultert, die an sich in fast allen (antiken und modernen) Staaten zu den Kernaufgaben der staatlichen Autorität zählt: die Aufrechterhaltung der öffentlichen Sicherheit mit polizeilichen Mitteln.

Der Fall von Bubon stellt aber offensichtlich kein singuläres Ereignis dar, sondern wie die juristische Literatur dieser Epoche andeutet, dürfte es sich hier eher um den Regelfall der lokalen Sicherheitsarbeit und nicht um die Ausnahme gehandelt haben. Dies wird deutlich, wenn der Jurist Marcianus im liber 2 seines Werkes ,De iudiciis publicis' problemlos auf ein edictum zurückgreifen kann, das der spätere Kaiser Antoninus Pius während seiner Amtszeit als proconsul Asiae (135/136?) verkündet hatte. 4 (see also pp. 153-154).

3 Fr. Schindler, Die Inschriften von Bubon, Nordlykien, SBAW 278 III, Wien 1972, 12-23 Nr. $2=$ Bull. épigr. 1973, 451 = AE 1979, 624 = Freis 111. Die von mir verwendete Übersetzung ist die von H. Freis, Historische Inschriften zur römischen Kaiserzeit von Augustus bis Konstantin (Darmstadt 1984) (Texte zur Forschung 49), Nr. 111.

4 Vgl. dazu A. Nogrady, Römisches Strafrecht nach Ulpian. Buch 7 bis 9 De officio proconsulis (Berlin 2006) (Freiburger rechtshistorische Abhandlungen Neue Folge 52), $28 \mathrm{ff}$. 
Sed et caput mandatorum exstat, quod divus Pius, cum provinciae Asiae praeerat, sub edicto proposuit, ut irenarchae, cum adprehenderint latrones, interrogent eos de sociis et receptatoribus et interrogationes litteris inclusas atque obsignatas ad cognitionem magistratus mittant. Igitur qui cum elogio mittuntur, ex integro audiendi sunt, etsi per litteras missi

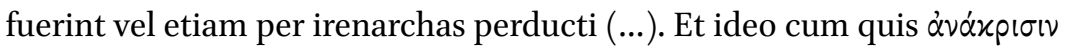
faceret, iuberi oportet venire irenarchen et, quod scripsit, exsequi; et si diligenter ac fideliter hoc fecerit conlaudandum eum. si parum prudenter non exquisitis argumentis, simpliciter denotare irenarchen minus rettulisse; sed si maligne interrogasse aut non dicta retulisse pro dictis eum comperit, ut vindicet in exemplum, ne quid et aliud postea tale facere moliatur.

Es existiert aber noch der Anfang der mandata, die der vergöttliche (Antoninus) Pius, als er die provincia Asia verwaltetete, in der Form eines Ediktes veröffentlichte. Demnach sollten die Irenarchen, wenn sie Übeltäter festgenommen hätten, diese über ihre Komplizen und Hintermänner ausfragen und die schriftlichen und unterzeichneten Verhörprotokolle zur Untersuchung durch den Magistraten senden. Folglich sollen diese (scil. die Übeltäter) mit dem Schreiben überstellten (Übeltäter), erneut gehört werden, obwohl sie zusammen mit einem Schreiben überstellt wurden oder sogar durch den Irenarchen (persönlich) überführt wurden (...). Und daher ist es notwendig, wenn jemand eine àváxpıбı durchführt, dass er den Irenarchen kommen lässt und das, was dieser geschrieben hat, überprüft. Und wenn er dies sorgfältig und getreulich gemacht hat, dann soll man ihn loben. Wenn er es weniger weise gemacht hat, dann soll man dies nicht in ausgewählten Formulierungen, sondern ganz einfach festhalten und den Irenarchen darauf hinweisen und sich weniger darauf beziehen. Wenn er aber feststellt, dass er allerdings etwas mit übler Absicht untersucht hat und etwas, was nicht ausgesagt wurde, als etwas gemeldet hat, was ausgesagt wurde, dann soll er ihn exemplarisch bestrafen, damit er es nicht wagt, später etwas anderes in dieser Art zu machen.

Dig. 48.3.6.1

Aus dem hier geschilderten und durchaus differenzierten Verfahren wird sehr deutlich, dass die Hauptlast der Polizeiarbeit ebenso wie die Bekämpfung der lokalen Unruhen von der römischen Provinzialverwaltung überhaupt nicht selbst verantwortet wurde, sondern freundlicherweise auf die Schultern der lokalen Verantwortlichen abgeladen worden war. Das hier beschriebene Verfahren war wohl ursprünglich, wie der Verweis auf das edictum des Antoninus 
Pius deutlich macht, lediglich für die provincia Asia eingeführt worden. Allerdings macht die Art und Weise, wie dieses edictum später von Marcianus in seiner eigenen Erörterung eingeführt wurde, deutlich, dass wir hier wahrscheinlich eine Art von Blaupause für die analoge Anwendung dieser Vorschriften auf andere Provinzen vor uns haben.

Damit beginnen allerdings die Schwierigkeiten, denn selbst die provinca Asia präsentierte sich keineswegs als ein homogenes Territorium, das durchgehend durch ein engmaschiges Netz von städtisch organisierten Gemeinden kontrolliert werden konnte. Denn es gab, abgesehen von den großen kaiserlichen und senatorischen Domänen, ${ }^{5}$ mit deren Existenz wir in dieser Weltgegend rechnen müssen, einige Bereiche im Binnenland, bei denen man vermuten darf, dass selbst in den spätesten Phasen der römischen Herrschaft der Grad der Urbanisierung und damit auch die damit einhergehende Kontrolle durch Sicherheitskräfte bestenfalls rudimentär gewesen sein dürfte. ${ }^{6}$ Die Sicherheitsprobleme in vielen anderen Regionen des Imperium Romanum, in denen es selbst nach Jahrhunderten nicht gelungen war, eine an Städten orientierte staatliche Struktur zu entwickeln, soll nur kurz erwähnt werden. ${ }^{7}$

Aber selbst die Existenz von Städten garantierte nicht unbedingt für Sicherheit. Dies beweist der berühmte Brief des Augustus an die Gemeinde Knidos (see also pp. 129-130). Hier wird deutlich, dass es den lokalen Autoritäten kaum möglich war, starke lokale Kräfte mit ihren Eigeninteressen wirksam unter Kontrolle zu halten. ${ }^{8}$ Man kann dem in einer Inschrift überlieferten Text dieses Briefes entnehmen, dass es offensichtlich zwischen zwei angesehenen Fami-

5 Vgl. u.a. Th. Corsten, Bauern und Bürger. Einflußmöglichkeiten von Landbesitzern auf das städtische Leben des kaiserzeitlichen Kleinasiens, in:F. Lerouxel, A.-V. Pont (Edd.), Propriétaires et citoyens dans l'Orient romain (Bordeaux 2016) (Ausonius 84), 261-273. M. Christol, Les domaines de Claudii Severi en Asie Mineure, in: F. Lerouxel, A.-V. Pont (Edd.), Propriétaires et citoyens dans l'Orient romain (Bordeaux 2016) (Ausonius 84), 275-287.

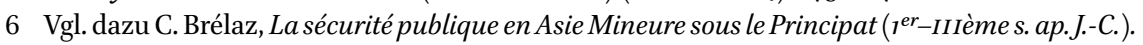
Institutions municipales et institutions impériales dans l'Orient romain (Basel 2005) (Schweizerische Beiträge zur Altertumsiwssenschaft 32) $5^{2} \mathrm{ff}$. Für die allgemeine Entwicklung vgl. S. Mitchell, Anatolia. Land, Men, and Gods in Asia Minor I. The Celts in Anatolia and the Impact of Roman Rule (Oxford 1993). Ebenso für die Motive, römische coloniae veteranorum anzulegen: B. Levick, Roman Colonies in Southern Asia Minor (Oxford 1967), 21 ff. Für die ländliche Unsicherheit vgl. R. MacMullen, The Enemies of the Roman Order. Treason, Unrest, and Alienation in the Empire (London, New York 1992), 255-268.

7 Dazu P. Herz, Das Entstehen einer Provinz. Gedanken zum römischen Recht und zur römischen Politik, in: U. Lohner-Urban, P. Scherrer (Hrsg.), Der obere Donauraum 50 v. Chr. bis $50 \mathrm{n}$. Chr. (Berlin 2015), 185-197.

8 R.T. Sherk, Roman Documents from the Greek East. Senatus Consulta and Epistulae to the Age of Augustus, (Baltimore 1969), 341-345 Nr. 67. 
lien dieser Gemeinde zu heftigen Streitigkeiten gekommen war. Im Verlauf dieses Streites hatten die beiden Söhne der einen Familie versucht, das Haus ihres Gegners zu stürmen, wobei einer der Söhne ums Leben gekommen war. Obwohl dies offenkundig durch einen Akt der Selbstverteidigung geschehen war, war die Familie des Getöteten nicht bereit gewesen, dies so einfach zu akzeptieren, und hatte versucht, diese Tat als Mord durch ein Gericht ahnden zu lassen. ${ }^{9}$ Selbst als ein Vertreter des Augustus die Angelegenheit persönlich untersucht hatte, hatten die Verwandten des Getöteten nicht aufgegeben und hatten das Verfahren bis zu einer Entscheidung in letzter Instanz, d.h. durch Augustus selbst, vorangetrieben.

Aus diesem Verfahren wird deutlich, dass die lokalen Kontrollmechanismen spätestens dann nicht mehr funktionieren konnten, wenn die Vertreter der Führungsschicht untereinander zerstritten waren oder deren privaten Interessen wie in diesem Fall unmittelbar betroffen waren. Spätestens dann war die Funktionsfähigkeit dieser Gemeinde ernsthaft in Frage gestellt.

Diese hier erkennbaren strukturellen Defizite waren den Verantwortlichen in Rom sicherlich bekannt. Sie haben allerdings die römische Zentralregierung zu keiner Zeit daran gehindert, an ihre lokalen Repräsentanten Erwartungen zu richten, die nur wenig an der Realität vor Ort orientiert waren. Deutlich wird dies in der Einleitung des Werkes ,De officio proconsulis', in der der Jurist Ulpianus am Beispiel der Aufgaben eines Provinzstatthalters die Grundzüge des römischen Herrschaftsmodels erläutert. ${ }^{10}$

congruit bono et gravi praesidi curare, ut pacata atque quieta provincia sit quam regit

es geziemt sich für einen guten und respektablen Statthalter, Sorge dafür zu tragen, daß die Provinz, die er regiert, befriedet und ruhig ist.

Dig. 1.18.13 pr. (lib. vii off. procos.)

Wenn man Ulpianus Glauben schenken möchte, dann war ein solcher Zustand für einen in seinem Sinne guten Provinzstatthalter recht leicht zu erreichen, denn er fährt dann fort:

quod non difficile obtinebit, si sollicite agat, ut malis hominibus provincia careat eosque conquirat: nam et sacrilegos, latrones, plagiarios, fures conausgeübt werden, sondern fiel natürlich in die Kompetenz des proconsul Asiae. 
quirere debet et prout quisque deliquerit in eum animadvertere, receptores eorum coercere, sine quibus latro duitius latere non potest.

dies wird er ohne Schwierigkeit erreichen (können), wenn er so handelt, dass die Provinz keine schlechten Menschen hat und er diese aufspürt. Denn er mussß die Tempelschänder, die Übeltäter, die Menschenräuber und die Diebe festnehmen und aburteilen, so wie es jeder nach seinem Vergehen verdient, und ihre Hintermänner in den Griff bekommen, ohne die sich kein Übeltäter längere Zeit verbergen kann. ${ }^{11}$

Diese sehr allgemein gehaltenen Staatsziele finden sich auch noch in zwei weiteren juristischen Quellen aus der severischen Periode ausformuliert. So kann Marcianus im 14. Buch seiner institutiones festhalten:

Mandatis autem cavetur de sacrilegiis, ut praesides sacrilegos latrones plagiarios conquirant et ut, prout quisque deliquerit, in eum anmadvertant.

In den Anweisungen wird über die Schänder von Heiligtümern bestimmt, dass die Provinzstatthalter die Tempelschänder, die Räuber und die Menschenräuber ermitteln und über sie ein Urteil fallen sollen, so wie es jeder von ihnen verdient.

Dig. 48.13 .4 .2

In noch knapperer Form findet sich dieser Grundsatz bei Paulus ausformuliert (liber xiii ad Sabinum).

Nam et in mandatis principum est, ut curet is, qui provinciae praeest, malis hominibus provinciam purgare ...

Denn auch in den Dienstanweisungen (mandata) der Kaiser steht, dass derjenige, der eine Provinz führt, (diese) Provinz von schlechten Menschen reinigen soll.

Dig. 1.18.3

11 Mit, coercere wird ein wesentlicher Bestandteil der statthalterlichen Befehlsgewalt angesprochen, also das Recht, zur Durchsetzung eines Befehls Zwangsmittel einsetzen zu dürfen (ius coercitionis). 
Wir dürfen wohl davon ausgehen, dass der hier deutlich gewordene Widerspruch zwischen dem sehr optimistisch formulierten Katalog an Anforderungen und der Realität nicht nur einem modernen Historiker, sondern auch den damals verantwortlichen Personen ins Auge fiel. Wie ist man also vorgegangen, um zumindest ein gewisses Äquilibrium zwischen einem solchen Anspruch und seiner Realisierung zu erreichen?

Einen gewissen Einblick liefert dazu ein sehr umfangreicher titulus im Corpus Iuris Civilis, der der ,Lex Iulia de vi publica' gewidmet ist. ${ }^{12}$ Dieser Abschnitt ist unter zwei Aspekten bemerkenswert. Er macht uns zunächst mit einer sehr großen Bandbreite an Straftatbeständen vertraut, die allesamt regelmäßig mit dem Einsatz von physischer Gewalt verbunden waren. Zur gleichen Zeit offenbart er auch etwas, was man als administrativen Generalverdacht gegenüber den eigenen Untertanen bezeichnen könnte. Offensichtlich hegten die römischen Autoritäten grundsätzlich den Verdacht, dass ihre Untertanen kaum bereit waren, ihre eigenen Streitigkeiten friedlich, d.h. etwa in den Formen eines regulären Gerichtsverfahrens, beizulegen, sondern eher dazu tendierten, gleich zu den Mitteln des Faustrechtes zu greifen. Man musste also auf Seiten der römischen Verwaltung davon ausgehen, dass es unmöglich sein würde, eine im Sinne der römischen Staates, friedliche“ Lebenssituation herzustellen, ohne gleichzeitig für die Missachtung der Regeln die schwersten Strafen anzudrohen.

Diese Einstellung wird besonders deutlich, wenn man etwa die Ausführungen des Juristen Marcianus, also eines Vertreters der severischen Zeit, zum Besitz und Einsatz von Waffen prüft.

Lege Iulia de vi publica tenetur, qui arma tela domi suae agrover inve villa praeter usum venationis vel itineris vel navigationis coegerit.

Nach der lex Iulia über die öffentliche Gewalt wird derjenige belangt, der Schutz- und Trutzwaffen in seinem Haus oder auf seinem Land oder in seinem Landgut hinausgehend über den Gebrauch bei der Jagd oder auf der Reise oder für die Seefahrt angesammelt hat.

Dig. 48.6.1 (Marcianus libro xiv institutionum)

Neben dem wahrscheinlich eher theoretisch bleibenden Versuch, neben wenigen genau spezifizierten Ausnahmen bereits den bloßen Besitz von Waffen grundsätzlich unter eine Strafandrohung zu stellen, ist dabei interessant, dass 
man auf Seiten der Juristen bei vielen Tatbeständen des Strafrechtes fast wie selbstverständlich davon ausging, dass bei einer solchen Gelegenheit sofort Waffen oder etwas allgemeiner gesprochen, physische Gewalt zum Einsatz kommen könnte. Dabei scheint der Gesetzgeber nicht nur an Waffen im klassischen Sinne gedacht zu haben, sondern wie eine Stelle aus den Paulus-Sentenzen (liber $v$ de sententiis) deutlich macht, dachte man dabei an alle Gegenstände, mit denen ein dazu entschlossener Mensch einem anderen Menschen körperlichen Schaden zufügen konnte.

Telorum autem appellatione omnia, ex quibus singuli homines nocere possunt, accipiuntur.

Unter der Bezeichnung ,tela' wird alles verstanden, mit dem einzelne Menschen Schaden zufügen können.

Dig. 48.6.11.1

Die Grenzen zwischen einer heftigen verbalen Auseinandersetzung und der direkten Drohung, physische Gewalt einzusetzen, scheinen in der Realität sehr fließend gewesen zu sein. Dies galt offensichtlich nicht nur für Auseinandersetzungen zwischen zivilen Parteien, sondern es hat ganz den Anschein, dass selbst die Vertreter der staatlichen Autorität unter der ständigen Bedrohung leben mussten, Opfer von offener Gewalt zu werden.

Denn anders lässt sich kaum die staatliche Drohung verstehen, durch die sich bereits die Behinderung eines Gerichtsverfahrens oder generell die Behinderung eines Amtsträgers bei der Ausübung seiner Amtspflichten zu einer Anklage nach der lex Iulia de vi publica auswachsen konnte. Ulpianus hat dazu einen ausführlichen Katalog an möglichen Delikten in seinem EdiktKommentar überliefert (liber lxviii ad edictum).

Qui dolo malo fecerit, quo minus iudicia tuto exercantur aut iudices ut oportet iudicent vel is, qui potestas imperiumve habebit, quam ei ius erit, decernat imperet faciat; qui ludos pecuniamve ab aliquo invite polliceri publice privatimve per iniuraim exegerit; item qui cum telo dolo malo in contione fuerit aut ubi iudicium publice exercebitur. Exceptus est, qui propter venationem habeat homines, qui cum bestiis pugnent, ministros ad ea habere conceditur.

Wer mit schlechtem Vorsatz bewirkt, dass weniger sicher Urteile vollstreckt werden oder Richter, so wie es notwendig ist, recht sprechen, oder dass derjenige, der eine Amtsgewalt (potestas) oder ein Imperium besit- 
zen wird, wenn er das Recht $\langle$ dazu $\rangle$ besitzt, $\langle$ etwas $\rangle$ entscheidet, befiehlt oder macht.

Wer von jemand gegen dessen Willen durch Unrecht erzwingt, dass er öffentlich oder privat verspricht, dass er Spiele oder Geld geben werde.

Ausgenommen ist derjenige, der wegen (der Abhaltung) Tierhetzen Menschen besitzt, die mit wilden Tieren kämpfen. Zu diesem Zweck ist es erlaubt, $\langle$ solche $\rangle$ Diener zu haben.

(1) Hac lege tenetur et qui convocatis hominibus vim fecerit, quo quis verberetur et pulsetur.

Nach diesem Gesetz wird auch derjenige bestraft, der mit zusammengerufenen Menschen Gewalt ausübt, durch die jemand verprügelt oder gestoßen wird.

(2) Damnato di vi publica aqua et igni interdicitur.

Einer Person, die wegen öffentlicher Gewalt verurteilt wurde, wird der Gebrauch von Wasser und Feuer untersagt [d.h. er wird aus der römischen Bürgerschaft ausgestoßen = der Schuldige wurde hingerichtet].

Dig. 48.6.10

Unter diesen sehr strikten Rahmenbedingungen konnten viele damals gesellschaftlich durchaus akzeptierte soziale Verhaltensformen, die in einem modernen Strafrecht relativ leicht bestraft werden würden, sehr schnell dazu führen, dass man sich plötzlich mit einer Kapitalstrafe bedroht sah. Denn die Bestimmungen dieses Gesetzes waren so allgemein formuliert und wohl auch ganz bewusst so breit angelegt worden, dass bereits eine spontane, aber nicht genehmigte Zusammenrottung einer größeren Menschenmenge auf öffentlichen Plätzen völlig ausreichen sein konnte, um einen Straftatbestand zu konstituieren, bei dem die öffentliche Gewalt mit allen Mitteln bis hin zur Verhängung der Kapitalstrafe einschreiten konnte. ${ }^{13}$

13 Wie brutal die römischen Autoritäten in solchen Fällen vorgehen konnten, beweisen die massenhaft verhängten Todesurteile nach den Unruhen unter den Gracchen oder dem Volkstribunat des Saturninus. Vgl. dazu immer noch J. Ungern-Sternberg von Pürkel, Untersuchungen zum spätrepublikanischen Notstandsrecht. Senatusconsultum ultimum und hostis-Erklärung (München 1970), $29 \mathrm{ff}$. 
Suetonius überliefert dazu in seiner Vita des Tiberius einen bezeichnenden Vorfall aus der italischen Stadt Pollentia, der sogar zum Einsatz von regulären Truppen in dieser Gemeinde führte. ${ }^{14}$

cum Pollentia plebs funus cuiusdam primipilaris non prius ex foro misisset quam extorta pecunia per vim heredibus ad gladiatorium munus, cohortem ab urbe et aliam a Cotti regno adsimulata itineris causa detectis repente armis concinentibusque signis per diversas portas in oppidum immisit ac partem maiorem plebei ac decurionum in perpetua vincula coiecit.

Als einmal die Volksmasse von Pollentia nicht zugelassen hatte, daß sich der Leichenzug für einen Primipilaren eher vom Marktplatz in Bewegung setzen konnte, bis sie den Erben durch die Androhung von Gewalt Geld zur Veranstaltung eines Gladiatorenspiels abgetrotzt hatte, ließ er [scil Tiberius] eine Kohorte von Rom aus, eine andere aus dem Königreich des Cottius losmarschieren, ohne den Grund für den Marsch zu offenbaren. Plötzlich ließ er sie in voller Kampfbewaffnung und mit Signalen, die zum Angriff bliesen, durch die Tore in die Stadt einmarschieren. Einen großen Teil der Volksmasse und der Gemeinderäte ließ er auf Lebenszeit ins Gefängnis werfen.

Sueton Tib. 37.3

Ein solches Vorkommnis, wie es hier von Sueton beschrieben wird, hätte nach den Kriterien, die wir bei Ulpianus finden, problemlos als strafwürdiges Vergehen nach der lex Iulia de vi publica eingestuft werden können, d.h. es wäre im Prinzip jederzeit möglich gewesen, nicht nur eine lebenslängliche Gefängnisstrafe, sondern auch die Hinrichtung zumindest der Wortführer anzuordnen. Wahrscheinlich schreckte man aber etwas vor diesem sehr radikalen Schritt zurück, denn die Tatsache, dass offensichtlich nicht nur die plebs der Stadt, sondern auch ein Teil des Stadtrates von Pollentia in diese Affaire verwickelt gewesen war und auch deswegen anschließend bestraft wurde, macht deutlich, dass es bei dieser heftigen öffentlichen Auseinandersetzung wahrscheinlich um wesentlich mehr als nur um die Abhaltung von Spielen ging. Es steckte also wahrscheinlich deutlich mehr hinter diesem Vorfall als uns Suetonius berichten kann. Es ist dabei eine durchaus mögliche Variante, dass der unbekannte

14 H. Galsterer, ,Politik in römischen Städten. Die ,seditio des Jahres 59 n.Chr. in Pompeii', in: W. Eck, H. Galsterer, H. Wolff (Hrsg.), Studien zur antiken Sozialgeschichte. Festschrift Friedrich Vittinghoff (Köln, Wien 1980), 323-338. 
primipilaris, dessen feierliches Begräbnis von der aufgebrachten Volksmasse zunächst verhindert wurde, entweder zu seinen Lebzeiten oder in seinem Testament seiner Heimatgemeinde die Abhaltung von ludi gladiatorii versprochen hatte und sich seine Erben jetzt geweigert hatten, dieses Versprechen umzusetzen.

Die römische Zentralregierung war bei solchen Ausbrüchen lokaler Gewalt durchaus in der Lage, differenziert darauf zu reagieren. Dies beweisen zwei Ereignisse aus der Regierungszeit Kaiser Neros, von denen Tacitus berichtet. Das erste Ereignis betraf die Gemeinde Pompeii.

(1) Sub idem tempus levi contentione orta atrox caedes colonos Nucerinos Pompeianosque gladiatorio spectaculo, quod Livineius Rufus, quem motum senatu rettuli, edebat, quippe oppidana lascivia in vicem incessente probram dein saxa, postremo ferrum sumpsere. validiore Pompeianorum plebe, apud quos spectaculum edebatur, ergo deportati sunt in urbem multi e Nucerinis trunco per vulnera corpore, ac plerique liberorum aut parentum mortes deflebant.

(2) cuius re iudicium princeps senatui, senatus consulibus permisit, et rursus re ad patres relata, prohibiti publice in decem annos eius modi coetu Pompeiani collegiaque, quae contra leges instituereant, dissolute; Livineius et qui alii seditionem conciverant exilio multati sunt.

(1) Etwa um diese Zeit ereignete sich aus kleinen Streitigkeiten zwischen den Bürgern von Nuceria und Pompeii ein schreckliches Gemetzel und zwar bei einem Gladiatorenspiel, das Livineius Regulus, von dem ich berichtet habe, dass er aus dem Senat entfernt worden war, gab. Weil die städtische Unbeherrschtheit sich gegenseitig anheizte, griffen sie (erst) zu Beschimpfungen, dann zu Steinen, dann zu Waffen (ferrum), wobei die plebs der Pompeianer, bei denen dieses Schauspiel veranstaltet wurde, die Oberhand behielt. Daher sind viele von den Nucerinern, deren Körper durch Wunden entstellt waren, in die Stadt (Rom) gebracht worden und viele beweinten den Tod ihrer Kinder oder Eltern.

(2) Das Urteil in dieser Sache überließ der Kaiser dem Senat, der Senat den Konsuln. Und diese berichteten wiederum dem Senat. Den Pompeianern wurden auf zehn Jahre öffentliche Zusammenkünfte dieser Art untersagt und es wurden die Vereine aufgelöst, die sie gegen die Gesetze eingerichtet hatten. Livineius und die anderen, die diesen Aufstand angezettelt hatten, wurden mit der Exilierung bestraft.

Tac. Ann. 14.17 
Die Besonderheit des Vorgehens in diesem Fall und auch die vergleichsweise milden Strafen, immerhin waren ja Menschen getötet worden, wird noch deutlicher, wenn man das Verhalten des Kaisers mit seinem Verhalten aus dem Vorjahr vergleicht.

Isdem consulibus auditae Puteolanorum legationes, quas diversas ordo plebs ad senatum miserat, illi vim multitudinis, hi magistratuum et primi cuiusque avaritiam increpantes. eaque seditio ad saxa et minas ignium progressa ne $\mathrm{c}\langle$ aed $\rangle \mathrm{dem}$ et arma proliceret, C. Cassium adhibendo remedio delectus. quia severitatem eius non tolerabant, precante ipso ad Scribonios fratres ea cura transfertur, data cohorte praetoria, cuius terrore et paucorum supplicio rediit oppidanis concordia.

Unter denselben Konsuln wurden Gesandtschaften der Puteolani gehört, die Rat und Volk getrennt zum Senat entsandt hatten. Jene beklagten die Gewalt der Masse, jene die Habgier der Magistrate und der ersten (Bürger). Damit sich dieser Aufstand, die sich schon zum Steinewerfen und zur Drohung, Feuer zu legen, fortentwickelt hatte, nicht Mord und Totschlag hervorrufe, wurde C. Cassius ausgewählt, um Heilung zu bringen. Weil sie aber seine Strenge nicht ertragen konnten und weil er selbst darum bat, wurde diese Aufgabe auf die Gebrüder Scribonius übertragen. Diesen wurde eine Prätorianerkohorte mitgegeben. Durch die Furcht vor dieser und die Hinrichtung von einigen wenigen Leuten wurde den Stadtbewohnern die Eintracht wiedergegeben.

Tac. Ann. 13.48

Wir haben zwei Beispiele für schwere Unruhen in italischen Gemeinden und zur gleichen Zeit zwei völlig unterschiedliche Behandlungen für solche Probleme. In Pompeii war die höchste Strafe das Exil für die Verantwortlichen, obwohl es während der Unruhen eine ganze Reihe von Todesopfern gegeben hatte. In Puteoli hingegen wurden Todesurteile verhängt, obwohl die dortigen Unruhen noch im Anfangsstadium (Drohungen und Steinwürfe) gewesen waren. Wie können wir diese Diskrepanz in der Strafzumessung erklären und was sagt uns dies über die Praxis der römischen Strafgerichtsbarkeit?

Zunächst scheint dies dafür zu sprechen, dass ein römischer Richter einen relativ großen Ermessensspielraum bei der Frage hatte, wie er eine Straftat bewertete und welches Strafmaß er für angemessen hielt. Er war nicht gehalten, unbedingt eine bestimmte Strafe zu wählen, sondern er konnte sich innerhalb eines relativ großen Spielraumes entscheiden. 
Ein weiterer Punkt, der hier wahrscheinlich eine bedeutende Rolle spielte, dürfte der rechtliche Status der beteiligten Personen gewesen sein. In Pompeii handelte es sich bei allen Personen, die an den Unruhen beteiligt gewesen waren, eindeutig um römische Bürger, während wir in einer großen Hafenstadt wie Puteoli wahrscheinlich mit einem sehr hohen Anteil von Leuten peregrini iuris in der lokalen Bevölkerung rechnen müssen. Es gibt sehr viele Indizien für die Vermutung, dass die römische Strafjustiz bei der Bestrafung von Nichtrömern wesentlich weniger Bedenken hatte, zu der Höchststrafe zu greifen (see also pp. 222-223).

Ein weiterer Punkt, der hier wahrscheinlich eine wichtige Rolle spielte, betrifft die Zusammensetzung der an diesen Streitigkeiten beteiligten Parteien. In Puteoli richteten sich die Proteste der Bevölkerung gegen die Stadtregierung. Dies bedeutet, dass sie damit die etablierte staatliche Ordnung nicht nur in dieser Stadt in Frage stellten. Dies erklärt auch den massiven Einsatz von militärischer Gewalt durch die zentralen Institutionen des römischen Staates. Die Unruhen in Pompeii waren zwar bedauerlich gewesen, sie hatten sich aber nicht gegen staatliche Institutionen gerichtet. Diese sehr unterschiedliche Strafzumessung entspricht zwar nicht unbedingt den Normen unseres modernen Rechtsempfindens, nach dem vor dem Gesetz alle Menschen gleich sein sollten, doch dies war sicherlich nicht die damals vorherrschende Rechtsauffassung.

Der ausufernde Einsatz von Gewalt war nicht nur auf die Städte beschränkt, sondern er scheint auch durchaus im ländlichen Raum üblich gewesen zu sein, um etwa Streitigkeiten zwischen Nachbarn zu lösen. Dieses deuten die entsprechenden Zeugnisse bei Marcianus (liber xiv institutionum = Dig. 48.6.3.2 und 6) und Paulus an (liberv de sententiis = Dig. 48.6.11). Dabei scheinen die hier angesprochenen Personen Angehörige der ländlichen Oberschicht gewesen zu sein, die ihre Streitigkeiten auf diesem Weg lösten.

\section{Marcianus}

(2) In eadem causa est, qui pessimo exemplo convocatu seditione villas expugnaverit et cum telis et armis bona rapuerit.

(6) Eadem lege tenetur, qui hominibus armatis possessorem domo agrove suo aut navi sua deiecerit expugnaverit.

(2) „Unter dasselbe Gesetz fallen (auch) diejenigen, die in einem äußerst schlechten Beispiel bei einer Zusammenrottung oder bei einem Aufstand Landgüter erobern oder mit Waffengewalt Besitztümer rauben“.

(6) „Nach eben diesem Gesetz wird derjenige belangt, der mit bewaff- 
neten Männern einen Besitzer aus seinem Haus oder Land oder von seinem Schiff herauswirft oder vertreibt.“

Paulus

$\mathrm{Hi}$, qui aedes alienas aut villas expilaverint effregerint expugnaverint, si quid in turba cum telis fecerint, capite puniuntur.

„Diejenigen, die fremde Gebäude oder Landgüter plündern, aufbrechen oder erobern, werden mit der Todesstrafe bestraft, wenn sie dies in einer Gruppe mit Waffen tun sollten“.

An diesem Punkt unserer Diskussion müssen wir uns auch einmal den folgenden Fragen stellen: 1. Warum war das Gewaltpotential in der Gesellschaft des Imperium Romanum überhaupt so hoch? 2. Warum ließ sich dieses Gewaltpotential nur so schwer kontrollieren bzw. im Sinne der römischen Gesetzgeber in ,zivilisierte‘ Bahnen lenken? Ich habe dazu eine Reihe von Argumenten zusammengestellt, die in ihrer Summe mögliche Erklärungswege aufzeigen können.

1. Es existierte in dieser Zeit ein sehr hohes Niveau der sozialen und wirtschaftlichen Ungleichheit. ${ }^{15}$

2. Für die Masse der damaligen Bevölkerung galt, dass sie wahrscheinlich in der Regel unter höchst prekären Umständen ihr Leben fristen musste, d.h. diese Menschen lebten permanent knapp am Existenzminimum. ${ }^{16}$

3. Es gab in allen Schichten der Bevölkerung eine große latente Bereitschaft, vorhandene Meinungsverschiedenheiten in gewaltsamen Formen zu artikulieren und auszutragen. ${ }^{17}$

4. Es gab auf allen Ebenen der staatlichen Verwaltung (Einzelgemeinde, Provinz) nur ein sehr eingeschränktes Instrumentarium an Mitteln, um den staatlichen Anspruch auf Gewährleistung der öffentlichen Sicherheit wirklich durchsetzen zu können. ${ }^{18}$

15 Vgl. Etwa M. Prell, Armut im antiken Rom (Stuttgart 1997) (Beiträge zur Wirtschafts- und Sozialgeschichte 77).

16 B. Tenger, Die Verschuldung im römischen Ägypten (1.-2. Jh. n.Chr.) (St. Katharinen 1993) (Pharos 3). E. Schaub, Studien zur Lebenssituation der Bevölkerung Ägyptens als Ursache der Revolten unter römischer Herrschaft. 30 v.Chr. bis 300 n.Chr. (Rahden 2014) (Pharos 31).

17 Für Ägypten vgl. A.Z. Bryen, Violence in Roman Egypt. A Study in Legal Interpretation (Philadelphia 2013). Vgl. Auch M. Gaddis, There is No Crime for Those Who Have Christ. Religious Violence in the Christian Roman Empire (Berkeley, London, Los Angeles 2005).

18 W. Nippel, Public Order in Ancient Rome (Cambridge 1995). Ch.J. Fuhrmann, Policing 
5. Selbst nach Jahrhunderten der römischen Herrschaft befanden sich große Bereiche des Imperium Romanum immer noch in einem eher präurbanen Stadium einer kulturellen Entwicklung, d.h. dort lebte eine vorwiegend nomadische bzw. seminomadische Bevölkerung. In diesem Stadium der Entwicklung stellte der Einsatz von physischer Gewalt ein allgemein akzeptiertes Instrument dar, um Streitigkeiten auszutragen.

6. Es gab in der damaligen Gesellschaft latent schwere interne Spannungen zwischen den unterschiedlichsten ethnischen und/oder religiösen Gruppierungen, deren Konflikte in der Regel gewaltsam ausgetragen wurden. ${ }^{19}$

Wir können also durchaus konstatieren, dass es einen eklatanten Widerspruch zwischen dem offiziell vertretenen Anspruch, Sicherheit und Frieden für alle Untertanen zu garantieren, und den Möglichkeiten gab, diese Ziele auch wirklich zu realisieren. Ganz im Gegenteil sprechen viele Indizien für die Vermutung, dass es außerhalb der größeren Städte und der großen Verkehrsmagistralen eine große Sphäre gab, in der wir durchaus von einem Zustand der weitgehenden Rechtsfreiheit sprechen können.

Spätestens wenn die etablierte staatliche Ordnung durch Krisen geschwächt war, zeigt sich sehr deutlich, dass sich unter der polierten Oberfläche der ,Pax Romana viele Konflikte verbargen, die vorher lediglich kaschiert worden waren, jetzt aber nicht mehr mit den Mitteln eines rigorosen Strafrechts kontrolliert werden konnten. Dies wird erstmals während der Krisenjahre unter der Regierung von Marcus Aurelius erkennbar, als etwa der hochrangige Offizier Valerius Maximianus auf dem Südbalkan gegen den Hirtenstamm der Brisei vorgehen musste oder sich das latente Unruhepotential der einheimischen Bukoloi in Ägypten zeigte. ${ }^{20}$

the Roman Empire. Soldiers, Administration, and Public Order (Oxford 2012). E. Künzl, Achtung Lebensgefahr! Die Legende von der inneren Sicherheit im antiken Rom (Mainz 2016).

19 Für die Streitigkeiten etwa der Juden mit den Angehörigen anderer ethnischer bzw. religiöser Gruppen vgl. etwa die Ereignisse in Caesarea Maritima zu Beginn des großen jüdischen Aufstandes (Ios. BJ 2.266ff.) oder die ständigen Auseinandersetzungen zwischen Juden und Nichtjuden in Alexandria. Dazu vgl. Philons Schrift ,In Flaccum'. Für das jüdische Alexandria vgl. die Beiträge in T. Georges, F. Albrecht, R. Feldmeier (Hrsg.), Alexandria (Tübingen 2013).

20 Für Valerius Maximiaus vgl. AE. 1956, 124, Zeile 15 f.: praeposito vexillationibus et ad detrahen/dam Briseorum latronum manum in confinio Macedon(iae) et $T(h) \mathrm{rac}($ iae $)$. Für die Bukoloi vgl. K. Blouin, Triangular Landscapes. Environment, Society, and the Stae in Nile Delta under Roman Rule (Oxford 2013). 
Es waren aber keine externe Feinde des Reiches, die man hier bekämpfen musste, sondern Teile der einheimischen Bevölkerung, die man nicht länger unter Kontrolle halten konnte. Dies alles unterstreicht nachdrücklich, dass zwischen der offiziell propagierten Sicherheit und der Realität doch ein großer Unterschied bestand. 